\title{
Necessary Conditions for Minimax Control Problems of Second Order Elliptic Partial Differential Equations*
}

\author{
Jiongmin Yong \\ Department of Mathematics, Fudan University \\ Shanghai 200433, China
}

\begin{abstract}
In this paper, we study an optimal control problem with the state equation being a second order semilinear elliptic partial differential equation containing a distributed control. The control domain is not necessarily convex. The cost functional, which is to be minimized, is the essential supremum over the domain $\Omega$ of some function of the state and the control. Our main result is the Pontryagin type necessary conditions for the optimal control. Ekeland variational principle and the spike variation technique are used in proving our result.
\end{abstract}

Keywords. elliptic equation, minimax control problem, maximum principle.

AMS(MOS) classifications. 35J85, 49B22

\section{$\S 1$. Introduction.}

In this paper, we consider a controlled second order elliptic partial differential equation

$$
\left\{\begin{array}{l}
-\Delta y(x)=f(x, y(x), u(x)), \quad \text { in } \Omega, \\
\left.y\right|_{\partial \Omega}=0,
\end{array}\right.
$$

where $\Omega$ is a bounded region in $\mathbb{R}^{n}$ with a smooth boundary $\partial \Omega$ and $f: \Omega \times \mathbb{R} \times U \rightarrow \mathbb{R}$ is a given map satisfying some conditions. $U$ is a metric space in which the control variable $u(\cdot)$ takes values. Function $y(\cdot)$ is called the state of the system, which is to be controlled. Under certain conditions, for each measurable function $u(\cdot)$, problem (1.1) admits a unique

* This work was partially supported by the NSF of China under Grant 19131050 and the Fok Ying Tung Education foundation. Current address: IMA, University of Minnesota, Minneapolis, MN 55455. 
solution $y(\cdot) \equiv y(\cdot ; u(\cdot))$ in some function space, say $\mathcal{Y}$. Then, for a given set $Q \subseteq \mathcal{Y}$, we may consider a state constraint of the following type:

$$
y(\cdot ; u(\cdot)) \in \mathcal{Y}
$$

Next, we let $h: \Omega \times \mathbb{R} \times U \rightarrow \mathbb{R}$ and consider a cost functional

$$
J(u(\cdot))=\underset{x \in \Omega}{\operatorname{esssup}} h(x, y(x ; u(\cdot)), u(x))
$$

Then, our optimal control problem is to find a control $u(\cdot)$ such that the corresponding state $y(\cdot ; u(\cdot))$ satisfies $(1.1)-(1.2)$ and minimizes the cost functional (1.3).

One of the motivation of our problem is the following: Suppose we would like to control the state $y(\cdot)$, which is subject to $(1.1)-(1.2)$, so that the largest deviation of it form the desired one, say $z(\cdot)$, is minimized. In this case, we only need to take

$$
h(x, y, u)=|y-z(x)|^{2} .
$$

Since the problem is to minimize a "maximum", it is usually referred as minimax control problem. Similar problem for ordinary differential equations was studied by several authors, see $[2,3,14]$. The purpose of this work is to give Pontryagin type maximum principle for optimal controls of our problem. Since the cost functional is not smooth (in some sense), we adopt an idea from $[2,3]$ to approximate it by the $L^{p}$-norm of function $h(x, y(x), u(x))$ and then let $p \rightarrow \infty$. In order the Ekeland variational principle applies, we need the stability of the optimal cost value. Namely, we need to show that the optimal cost value of the approximating problem approaches that of the original one. Due to the state constraint, we have to impose a condition to ensure such a stability. On the other hand, since $U$ is merely a metric space, no convexity can be talked about. Thus, we use the spike variation technique as $[11,21]$ in deriving the maximum principle. We have proved the nontriviality of the Lagrange multiplier. We should admit that, at present time, we are only able to prove the result for the case $h$ has the form

$$
h(x, y, u)=g(x, y)+\ell(x, u), \quad \forall(x, y, u) \in \Omega \times \mathbb{R} \times U
$$

Our approach seems not applicable to general case. However, we conjecture that the result should hold for general $h$. We notice that in [2] (for ODE systems with no state constraint), 
same problem also exists and the proof provided there, for the case of general $h$, seems questionable to us. Also, the nontriviality of the Lagrange multiplier was not mentioned in [2]. Finally, we note that the proofs of [2] heavily relied on the dynamic programming and viscosity solutions for HJB equations. These techniques, however, are not applicable for elliptic systems here. Besides, unlike in [2], we will not use the epi-convergence. For the completeness, in this paper, we also present an existence result of optimal controls, whose proof is basically similar to that given in [20], which was for Lagrange type cost functional.

We refer the readers to $[4,14,15]$ for standard optimal control theory of finite dimensions, to $[1,5-7,11,12,18-21]$ for infinite dimensional counterpart.

To conclude this introduction, let us point out that our approach applies to general second order elliptic partial differential equations, namely, we may replace the Laplacian by a general second order elliptic operator with smooth coefficients. Also, we may consider quasilinear systems, boundary control problems as well as problems for parabolic equations. Some interesting extensions will appear elsewhere.

\section{$\S 2$. Preliminaries.}

Let us start with some assumptions which will be assumed throughout of the paper. Let $\Omega \subseteq \mathbb{R}^{n}$ be a bounded region with a smooth boundary $\partial \Omega$ and $U$ be a Polish space $([10])$. Let $f: \Omega \times \mathbb{R} \times U \rightarrow \mathbb{R}, g: \Omega \times \mathbb{R} \rightarrow \mathbb{R}$ and $\ell: \Omega \times U \rightarrow \mathbb{R}$ be given maps satisfying the following: ( $h$ is defined by (1.4))

(i) $f(x, y, u)$ and $g(x, y)$ are differentiable in $y$ and for some constant $L>0$,

$$
\begin{gathered}
0 \leq-f_{y}(x, y, u), \quad|f(x, y, u)| \leq L, \quad \forall(x, y, u) \in \Omega \times \mathbb{R} \times U, \\
\left|g_{y}(x, y)\right|, \quad|h(x, y, u)| \leq L, \quad \forall(x, y, u) \in \Omega \times \mathbb{R} \times U .
\end{gathered}
$$

(ii) Maps $f(x, y, u), f_{y}(x, y, u), g(x, y), g_{y}(x, y)$ and $\ell(x, u)$ are continuous on $\Omega \times \mathbb{R} \times U$.

Remark 2.1. The above conditions can be relaxed substantially, say, $f$ and $f_{y}$ are merely measurable in $x$, the boundedness of $f$ and $h$ is replaced by the linear growth in $y$, 
etc. We prefer not get into such a generality since the result will be similar and the main idea is the same.

Next, we define

$$
\mathcal{U}=\{u(\cdot): \Omega \rightarrow U \mid u(\cdot) \text { measurable }\}
$$

Any element $u(\cdot) \in \mathcal{U}$ is referred as a control. From $[9,17]$, we know that under the above (i), for any $u(\cdot) \in \mathcal{U}$, problem (1.1) admits a unique solution $y(\cdot) \equiv y(\cdot ; u(\cdot)) \in$ $W^{2, p}(\Omega) \bigcap W_{0}^{1, p}(\Omega)$, for all $p \geq 1$. Moreover, there exists a constant $C=C(p, L, \Omega)$ such that

$$
\|y(\cdot ; u(\cdot))\|_{W^{2, p}(\Omega)} \leq C, \quad \forall u(\cdot) \in \mathcal{U}
$$

Thus, by Sobolev embedding theorem, we have

$$
\|y(\cdot ; u(\cdot))\|_{C^{1, \alpha}(\bar{\Omega})} \leq C, \quad \forall u(\cdot) \in \mathcal{U},
$$

with the constant $C$ and $\alpha \in(0,1)$ being independent of $u(\cdot)$. Hereafter, $C$ will be generic constants which could be different in different places. Now, we let $p>n$ be fixed and let $\mathcal{Y}$ be a separable Banach space containing $W_{0}^{1, p}(\Omega)$ (topologically):

$$
\mathcal{Y} \supseteq W_{0}^{1, p}(\Omega)
$$

We let $Q \subseteq \mathcal{Y}$ be convex and closed. Then, by the above analysis, we see that for any $u(\cdot) \in \mathcal{U}$, we have

$$
y(\cdot ; u(\cdot)) \in \mathcal{Y}
$$

Thus, the state constraint (1.2) makes sense. It is important to notice that (1.2) includes many interesting cases. Let us point out some of them. We let $\mathcal{Y}=C(\bar{\Omega})$ and

$$
Q=\left\{y(\cdot) \in \mathcal{Y} \mid y\left(x_{i}\right)=a_{i}, \quad 1 \leq i \leq m\right\}
$$

for some $x_{i} \in \Omega, a_{i} \in \mathbb{R}, 1 \leq i \leq m$. Under this choice, (1.2) gives a pointwise constraint for the state. If we take $\mathcal{Y}=L^{p}(\Omega)$ and let

$$
Q=\left\{y(\cdot) \in \mathcal{Y} \mid y(x) \geq 0, \quad \text { a.e. } x \in \Omega, \quad \int_{\Omega} y(x) h(x) d x \leq 1\right\},
$$


for some $h(\cdot) \in L^{p^{\prime}}(\Omega)\left(p^{\prime}=p /(p-1)\right)$, then, (1.2) gives another interesting state constraint. Moreover, it is also possible to replace (2.5) by

$$
\mathcal{Y} \supseteq W^{2, p}(\Omega) \bigcap W_{0}^{1, p}(\Omega)
$$

Clearly, $(2.6)$ is still valid. Then, we take $\mathcal{Y}=C^{1}(\bar{\Omega})$ and let

$$
Q=\left\{y(\cdot) \in \mathcal{Y} \mid \nabla y\left(x_{i}\right)=b_{i}, \quad 1 \leq i \leq m\right\}
$$

for some $x_{i} \in \Omega, b_{i} \in \mathbb{R}^{n}, 1 \leq i \leq m$. Under this choice, (1.2) provides a pointwise constraint for the gradient of the state. Similar constraints like (2.7) and (2.10) for other types of optimal control problem were considered in $[5,7,21]$. We may cook up some other interesting examples of state constraints. In what follows, we will restrict ourselves to the case (2.5). The case (2.9) will not be treated in this paper. Some relevant results will appear elsewhere.

Of course, for any given $u(\cdot) \in \mathcal{U}$, the corresponding state $y(\cdot ; u(\cdot))$ does not necessarily satisfy the constraint (1.2). Thus, let us introduce

$$
\begin{aligned}
& \mathcal{A}=\{(y(\cdot), u(\cdot)) \in \mathcal{Y} \times \mathcal{U} \mid(y(\cdot), u(\cdot)) \text { satisfies }(1.1)\}, \\
& \mathcal{A}_{Q}=\{(y(\cdot), u(\cdot)) \in \mathcal{A} \mid y(\cdot) \in Q\} \\
& \mathcal{U}_{Q}=\left\{u(\cdot) \in \mathcal{U} \mid(y(\cdot ; u(\cdot)), u(\cdot)) \in \mathcal{A}_{Q}\right\} .
\end{aligned}
$$

Any element $(y(\cdot), u(\cdot)) \in \mathcal{A}_{Q}$ is referred as an admissible pair, and any $u(\cdot) \in \mathcal{U}_{Q}$ and the corresponding $y(\cdot ; u(\cdot))$ are called admissible control and state, respectively. The cost functional is defined as (1.3). Clearly, it is defined for all $u(\cdot) \in \mathcal{U}$. Our optimal control problem can be stated as follows.

Problem C. Find $\bar{u}(\cdot) \in \mathcal{U}_{Q}$, such that

$$
J(\bar{u}(\cdot))=\inf _{\mathcal{U}_{Q}} J(u(\cdot))
$$

Now, let us make some reductions. First of all, by scaling, we may assume

$$
|\Omega| \equiv \operatorname{meas} \Omega=1
$$


Next, we let

$$
\widehat{h}(x, y, u)=\frac{h(x, y, u)+L+1}{2(L+1)}, \quad \forall(x, y, u) \in \Omega \times \mathbb{R} \times U .
$$

Then, by (2.2), we know that

$$
0<\frac{1}{2(L+1)} \leq \widehat{h}(x, y, u) \leq \frac{2 L+1}{2(L+1)}<1, \quad \forall(x, y, u) \in \Omega \times \mathbb{R} \times U .
$$

On the other hand, it is clear that to minimize $J(u(\cdot))$ is equivalent to minimize

$$
\widehat{J}(u(\cdot)) \equiv \underset{x \in \Omega}{\operatorname{essup}} \widehat{h}(x, y(x ; u(\cdot)), u(x)) .
$$

Hence, without loss of generality, we may assume

$$
0<a \leq h(x, y, u) \leq b<1, \quad \forall(x, y, u) \in \Omega \times \mathbb{R} \times U
$$

We will keep assumptions (2.13) and (2.16) in the rest of the paper. Clearly, under this assumption,

$$
\underset{x \in \Omega}{\operatorname{esssup}} h(x, y(x), u(x))=\|h(\cdot, y(\cdot), u(\cdot))\|_{L^{\infty}(\Omega)}
$$

To conclude this section, let us present an existence result for the optimal controls. In this result, we do not need $h$ to be of form (1.4). Now, we define

$$
\begin{aligned}
\mathcal{E}(x, y)=\left\{\left(\lambda^{0}, \lambda\right)\right. & \in \mathbb{R} \times \mathbb{R}^{n} \mid \lambda^{0} \geq h(x, y, u), \\
\lambda & =f(x, y, u), \quad \text { for some } u \in U\} .
\end{aligned}
$$

Then, we have the following result.

Theorem 2.2. Let $\mathcal{E}(x, y)$ be convex and closed for each $(x, y) \in \Omega \times \mathbb{R}$. let $\mathcal{A}_{Q}$ be nonempty. Then, Problem $C$ admits at least one solution.

Proof. Let $\left\{u_{k}(\cdot)\right\}_{k \geq 1} \subset \mathcal{U}_{Q}$ be a minimizing sequence of Problem $\mathrm{C}$ and $y_{k}(\cdot) \equiv$ $y\left(\cdot ; u_{k}(\cdot)\right)$ be the corresponding states. Then, by $(2.3)$, we may assume that

$$
\begin{array}{ll}
y_{k}(\cdot) \stackrel{s}{\rightarrow} \widetilde{y}(\cdot), & \text { in } C(\bar{\Omega}), \\
y_{k}(\cdot) \stackrel{w}{\longrightarrow} \widetilde{y}(\cdot), & \text { in } W^{2, p}(\Omega) .
\end{array}
$$


We may also assume

$$
\begin{array}{ll}
f\left(\cdot, y_{k}(\cdot), u_{k}(\cdot)\right) \stackrel{w}{\longrightarrow} \tilde{f}(\cdot), & \text { in } L^{p}(\Omega), \\
h\left(\cdot, y_{k}(\cdot), u_{k}(\cdot)\right) \stackrel{w}{\longrightarrow} \widetilde{h}(\cdot), & \text { in } L^{p}(\Omega) .
\end{array}
$$

Thus, we have

$$
\left\{\begin{array}{l}
-\Delta \widetilde{y}(x)=\tilde{f}(x), \quad \text { in } \Omega \\
\left.\widetilde{y}\right|_{\partial \Omega}=0
\end{array}\right.
$$

On the other hand, by definition

$$
\left(h\left(x, y_{k}(x), u_{k}(x)\right), f\left(x, y_{k}(x), u_{k}(x)\right)\right) \in \mathcal{E}\left(x, y_{k}(x)\right), \quad \text { a.e. } x \in \Omega
$$

Then, by Mazur's theorem and (2.19)-(2.20), we obtain

$$
(\widetilde{h}(x), \tilde{f}(x)) \in \overline{\operatorname{co}} \mathcal{E}(x, \widetilde{y}(x))=\mathcal{E}(x, \widetilde{y}(x)), \quad \text { a.e. } x \in \Omega
$$

Thus, by a Filippov type theorem $([20,10])$, we can find a $\widetilde{u}(\cdot) \in \mathcal{U}$, such that

$$
\left\{\begin{array}{l}
\tilde{h}(x) \geq h(x, \tilde{y}(x), \tilde{u}(x)), \\
\tilde{f}(x)=f(x, \tilde{y}(x), \tilde{u}(x)),
\end{array} \quad \text { a.e. } x \in \Omega .\right.
$$

Clearly, $(\tilde{y}(\cdot), \tilde{u}(\cdot)) \in \mathcal{A}_{Q}$ is an optimal pair.

We see that the proof of the above result is very similar to those given in [20].

\section{$\S 3$. Necessary Conditions.}

In this section, we state the Pontryagin type necessary conditions for optimal controls of our Problem C. The proof will be carried out in sequel sections.

Now, we let $\bar{u}(\cdot) \in \mathcal{U}_{Q}$ be an optimal control and $\bar{y}(\cdot)$ be the corresponding state. We impose a further assumption.

(H) Let $\left(y_{k}(\cdot), u_{k}(\cdot)\right) \in \mathcal{A}$ satisfy

$$
\lim _{k \rightarrow \infty} d\left(y_{k}(\cdot), Q\right)=0
$$


where

$$
d(y(\cdot), Q)=\inf _{q(\cdot) \in Q}\|y(\cdot)-q(\cdot)\| \mathcal{y}
$$

Then,

$$
\varliminf_{k \rightarrow \infty} J\left(u_{k}(\cdot)\right) \geq \bar{m} \equiv \inf _{\mathcal{U}_{Q}} J(u(\cdot))
$$

This assumption seems crucial in our approach. We do not know if one can remove this condition. Similar condition was used by the author in [19] for treating nonsmooth problem. We will make some remarks on this assumption a little later. Next, for any $u(\cdot) \in \mathcal{U}$, we introduce the variational system associated with $(\bar{y}(\cdot), \bar{u}(\cdot))$ as follows:

$$
\left\{\begin{aligned}
-\Delta z(x) & =f_{y}(x, \bar{y}(x), \bar{u}(x)) \\
& +f(x, \bar{y}(x), u(x))-f(x, \bar{y}(x), \bar{u}(x)), \quad \text { in } \Omega, \\
\left.z\right|_{\partial \Omega}=0 . &
\end{aligned}\right.
$$

Clearly, under our assumptions, for any $u(\cdot) \in \mathcal{U}$, there exists a unique solution $z(\cdot ; u(\cdot)) \in$ $\mathcal{Y}$ of $(3.3)$. We define

$$
\mathcal{R}=\{z(\cdot ; u(\cdot)) \mid u(\cdot) \in \mathcal{U}\}
$$

This is called the reachable set of the variational system (3.3). It is clear that $0 \in \mathcal{R}$. We say that $Q \subset \mathcal{Y}$ is finite codimensional in $\mathcal{Y}$ if there exists a point $z \in Q$, such that $\operatorname{span}\{Q-z\}$ is a finite codimensional subspace in $\mathcal{Y}$ and the relative interior of $\overline{\operatorname{co}}\{Q-z\}$ in $\operatorname{span}\{Q-z\}$ is nonempty. It is not hard to see that in the definition of codimensionality, the choice of the point $z \in Q$ is irrelevant. Now, let us state our main result of this paper. Theorem 3.1. Let $(\bar{y}(\cdot), \bar{u}(\cdot)) \in \mathcal{A}_{Q}$ be optimal and let $(H)$ hold. Moreover, let $Q$ be finite codimensional in $\mathcal{Y}$. Then, there exist $\left(\psi^{0}, \varphi\right) \in[-1,0] \times \mathcal{Y}^{*} \backslash\{0\}, \mu \in\left(L^{\infty}(\Omega)\right)^{*} \backslash\{0\}$ and $\psi(\cdot) \in W_{0}^{1, p^{\prime}}(\Omega), p^{\prime}=p /(p-1) \in(1, n /(n-1)),\left(\psi^{0}, \psi(\cdot)\right) \neq 0$, such that

$$
\begin{gathered}
\left\{\begin{aligned}
-\Delta \psi & =f_{y}(x, \bar{y}(x), \bar{u}(x)) \psi(x)+\psi^{0} g_{y}(x, \bar{y}(x)) \mu+\varphi, \\
\psi & \left.\right|_{\partial \Omega}=0,
\end{aligned}\right. \\
\operatorname{supp} \mu \subseteq\left\{x \in \Omega \mid h(x, \bar{y}(x), \bar{u}(x))=\|h(\cdot, \bar{y}(\cdot), \bar{u}(\cdot))\|_{L^{\infty}(\Omega)}\right\},
\end{gathered}
$$




$$
\begin{gathered}
\mu(\Omega) \equiv\left\langle\mu, \chi_{\Omega}\right\rangle \geq a, \\
\langle\varphi, q(\cdot)-\bar{y}(\cdot)\rangle \leq 0, \quad \forall q(\cdot) \in Q, \\
\psi(x) f(x, \bar{y}(x), \bar{u}(x))=\max _{u \in U(x)} \psi(x) f(x, \bar{y}(x), u), \quad \text { a.e. } x \in \bar{\Omega}_{0},
\end{gathered}
$$

where

$$
\begin{gathered}
\Omega_{0}=\left\{x \in \Omega \mid h\left(x, \bar{y}(x), \bar{u}(x)<\|h(\cdot, \bar{y}(\cdot), \bar{u}(\cdot))\|_{L^{\infty}(\Omega)}\right\},\right. \\
U(x)=\left\{u \in U \mid h(x, \bar{y}(x), u) \leq\|h(\cdot, \bar{y}(\cdot), \bar{u}(\cdot))\|_{L^{\infty}(\Omega)}\right\}, \quad x \in \Omega .
\end{gathered}
$$

In the case that $\ell(x, u) \equiv 0, \Omega_{0}$ can be replaced by $\Omega, U(x)=U$ and $U$ can be any metric space.

In the above, we refer to (3.5) as the adjoint system, which is understood as an obvious weak (or variational) sense. Condition (3.6) is understood as the following: For any $S \subset \Omega_{0}$,

$$
\mu(S) \equiv\left\langle\mu, \chi_{S}\right\rangle=0
$$

We refer to (3.8) as the transversality condition and (3.9) as the maximum condition. We see that if $\psi(\cdot) \neq 0$, then, (3.9) gives a necessary condition for the optimal control $\bar{u}(\cdot)$. Whereas, if $\psi(\cdot)=0$, then, (3.5) tells us that

$$
\psi^{0} g_{y}(x, \bar{y}(x)) \mu+\varphi=0 .
$$

This implicitly gives a necessary condition for $\bar{u}(\cdot)$. By $\left(\psi^{0}, \varphi\right) \neq 0$, we know that (3.13) is a nontrivial condition. Also, we should note in the case $h$ does depend on $u$ and

$$
h(x, \bar{y}(x), \bar{u}(x))=\|h(\cdot, \bar{y}(\cdot), \bar{u}(\cdot))\|_{L^{\infty}(\Omega)}, \quad \text { a.e. } x \in \Omega,
$$

one has meas $\Omega_{0}=0$. When this happens, (3.9) does not tell us anything. But (3.14) has already given us something. 
To conclude this section, let us make some comments on $(\mathrm{H})$. First of all, if $Q=\mathcal{Y}$, i.e., there is no state constraint, then, $(\mathrm{H})$ holds. Secondly, if for each $(x, y) \in \Omega \times \mathbb{R}$, the set $f(x, y, U)$ is convex and closed, and $h(x, y, u) \equiv h(x, y)$, then, $(\mathrm{H})$ holds. In fact, in this case, if $\left(y_{k}(\cdot), u_{k}(\cdot)\right) \in \mathcal{A}$ satisfying (3.1), then, we can show that there exists a pair $(\widetilde{y}(\cdot), \widetilde{u}(\cdot)) \in \mathcal{A}_{Q}$, such that for some subsequence,

$$
\left\|y_{k}(\cdot)-\widetilde{y}(\cdot)\right\|_{W^{1, p}(\Omega)} \rightarrow 0 .
$$

Thus,

$$
\bar{m} \leq J(\widetilde{u}(\cdot)) \leq \lim _{k \rightarrow \infty} J\left(u_{k}(\cdot)\right) .
$$

It is not hard to see that actually, if the conditions of Theorem 2.2 hold, then, $(\mathrm{H})$ holds. Hence, assumption $(\mathrm{H})$ is very general.

\section{§. Approximation of the Control Problem.}

Let us first give several results for elliptic equations.

Lemma 4.1. Let $c(\cdot) \in L^{\infty}(\Omega), c(x) \leq 0$. Then, for any $r>n$, there exists a constant $C=C\left(r,\|c(\cdot)\|_{L^{\infty}}, \Omega\right)$, such that for any solution $z(\cdot)$ of

$$
\left\{\begin{aligned}
-\Delta z(x) & =c(x) z(x)+\tilde{g}(x), \quad \text { in } \Omega, \\
\left.z\right|_{\partial \Omega} & =0
\end{aligned}\right.
$$

with $\widetilde{g}(\cdot) \in L^{r}(\Omega)$, it holds

$$
\|z(\cdot)\|_{W^{2, r}(\Omega)} \leq C\|\widetilde{g}(\cdot)\|_{L^{r}(\Omega)} .
$$

Proof. First, by $[9,13,16]$, we know that

$$
\|z(\cdot)\|_{L^{\infty}(\Omega)} \leq C\|\tilde{g}(\cdot)\|_{L^{r}(\Omega)},
$$

with $C=C\left(r,\|c\|_{L^{\infty}}, \Omega\right)$. Then, by standard $L^{p}$-estimate, we obtain

$$
\begin{aligned}
\|z(\cdot)\|_{W^{2, r}(\Omega)} & \leq C\left(\|c(\cdot) z(\cdot)\|_{L^{r}(\Omega)}+\|\tilde{g}(\cdot)\|_{L^{r}(\Omega)}\right) \\
& \leq C\left(\|z(\cdot)\|_{L^{\infty}(\Omega)}+\|\widetilde{g}(\cdot)\|_{L^{r}(\Omega)}\right) \leq C\|\widetilde{g}(\cdot)\|_{L^{r}(\Omega)} .
\end{aligned}
$$


This proves $(4.2)$.

Corollary 4.2. Let $u(\cdot), \widehat{u}(\cdot) \in \mathcal{U}$ and

$$
E=\{x \in \Omega \mid u(x) \neq \widehat{u}(x)\}
$$

Let $y(\cdot)$ and $\widehat{y}(\cdot)$ be the states corresponding to $u(\cdot)$ and $\widehat{u}(\cdot)$, respectively. Then,

$$
\|y(\cdot)-\widehat{y}(\cdot)\|_{W^{2, r}(\Omega)} \leq C|E|^{1 / r}
$$

where $C$ is independent of $u(\cdot)$ and $\widehat{u}(\cdot),|E|=$ meas $E$.

Proof. Let $z(\cdot)=y(\cdot)-\widehat{y}(\cdot)$. Then, $z(\cdot)$ satisfies

$$
\begin{aligned}
-\Delta z(x)= & f(x, y(x), u(x))-f(x, \widehat{y}(x), \widehat{u}(x)) \\
= & \int_{0}^{1} f_{y}(x, \widehat{y}(x)+\theta z(x), \widehat{u}(x)) d \theta z(x) \\
& \quad+[f(x, y(x), u(x))-f(x, y(x), \widehat{u}(x))] \chi_{E}(x) .
\end{aligned}
$$

Thus, by Lemma 4.1, we obtain

$$
\begin{aligned}
\|z(\cdot)\|_{W^{2, r}(\Omega)} & \leq C\left\|[f(\cdot, y(\cdot), u(\cdot))-f(\cdot, y(\cdot), \widehat{u}(\cdot))] \chi_{E}(\cdot)\right\|_{L^{r}(\Omega)} \\
& \leq C|E|^{1 / r} .
\end{aligned}
$$

This gives (4.6).

Now, let $r \geq 1$. We define

$$
J_{r}(u(\cdot))=\left(\int_{\Omega} h(x, y(x ; u(\cdot)), u(x))^{r} d x\right)^{1 / r}, \quad \forall u(\cdot) \in \mathcal{U}
$$

and let

$$
m_{r}=\inf _{u(\cdot) \in \mathcal{U}_{Q}} J_{r}(u(\cdot))
$$

The following result is the stability of the optimal cost value. This result is crucial in sequel. Also, we will find that $h$ does not have to be of form (1.4) in this result.

Theorem 4.3. Let $(H)$ hold. Then, it holds that

$$
\lim _{r \rightarrow \infty} m_{r}=\bar{m} \equiv \inf _{\mathcal{U}_{Q}} J(u(\cdot))
$$


To prove this result, we need the following lemmas.

Lemma 4.4. There exists a nondecreasing continuous function $\widehat{\omega}:[0, \infty) \rightarrow[0, \infty)$ such that for any $(y(\cdot), u(\cdot)) \in \mathcal{A}$ and $\alpha \in \mathbb{R}$, there exists a $(\widetilde{y}(\cdot), \widetilde{u}(\cdot)) \in \mathcal{A}$, such that

$$
h(x, \tilde{y}(x), \tilde{u}(x)) \leq \alpha+\widehat{\omega}\left(\left|M_{\alpha}\right|\right), \quad \text { a.e. } x \in \Omega
$$

where

$$
M_{\alpha}=\{x \in \Omega \mid h(x, y(x), u(x))>\alpha\}
$$

and

$$
E \equiv\{x \in \Omega \mid u(x) \neq \widetilde{u}(x)\} \subseteq M_{\alpha}
$$

Proof. If $\left|M_{\alpha}\right|=0$ or $\left|M_{\alpha}\right|=1$ (recall $|\Omega|=1$ ), (4.12) is trivially true. Thus, we let $0<\left|M_{\alpha}\right|<1$. Let $\delta>0$ be such that

$$
\left|M_{\alpha}\right|<\left|\mathcal{O}_{\delta}(0)\right|<2\left|M_{\alpha}\right|
$$

where $\mathcal{O}_{\delta}(x)$ is the open ball centered at $x$ with radius $\delta$. Then, we can choose $x_{i} \in \Omega$, such that

$$
\bigcup_{i \geq 1} \mathcal{O}_{\delta}\left(x_{i}\right) \supset \Omega
$$

By (4.15), we know that for each $i \geq 1$, there exists an $\widetilde{x}_{i} \in \mathcal{O}_{\delta}\left(x_{i}\right) \backslash M_{\alpha}$. For such $\widetilde{x}_{i}$, we know that

$$
h\left(\widetilde{x}_{i}, y\left(\tilde{x}_{i}\right), u\left(\widetilde{x}_{i}\right)\right) \leq \alpha
$$

Then, we define

$$
\tilde{u}(x)= \begin{cases}u(x), & x \in \Omega \backslash M_{a} \\ u\left(\widetilde{x}_{i}\right), & x \in\left[\mathcal{O}_{\delta}\left(x_{i}\right) \backslash \bigcup_{j=1}^{i-1} \mathcal{O}_{\delta}\left(x_{j}\right)\right] \cap M_{\alpha}\end{cases}
$$

Clearly, (4.14) holds. By Corollary 4.2, we know that there exists a constant $C$, independent of $u(\cdot)$ and $\tilde{u}(\cdot)$, such that $(\tilde{y}(\cdot)=y(\cdot ; \tilde{u}(\cdot)))$

$$
\| y(\cdot)-\left.\widetilde{y}(\cdot)\right|_{W^{2, p}(\Omega)} \leq C|E|^{1 / p} \leq C\left|M_{\alpha}\right|^{1 / p}
$$


Now, for any $x \in \Omega \backslash M_{\alpha}$, we have (let $\omega_{h}(\cdot)$ be the modulus of continuity for $h$ )

$$
\begin{aligned}
& h(x, \tilde{y}(x), \tilde{u}(x))=h(x, \widetilde{y}(x), u(x)) \\
& \leq \alpha+\omega_{h}(|y(x)-\widetilde{y}(x)|) \leq \alpha+\omega_{h}\left(C\left|M_{\alpha}\right|^{1 / p}\right) .
\end{aligned}
$$

For $x \in M_{\alpha} \bigcap\left[\mathcal{O}_{\delta}\left(x_{i}\right) \backslash \bigcup_{j=1}^{i-1} \mathcal{O}_{\delta}\left(x_{j}\right)\right]$,

$$
\begin{aligned}
h(x, \widetilde{y}(x), \widetilde{u}(x)) & =h\left(x, \widetilde{y}(x), u\left(\widetilde{x}_{i}\right)\right) \\
& \leq \alpha+\omega_{h}\left(\left|x-\widetilde{x}_{i}\right|+|y(x)-\widetilde{y}(x)|+\left|y\left(\widetilde{x}_{i}\right)-y(x)\right|\right) \\
& \leq \alpha+\omega_{h}\left(\delta+C\left|M_{\alpha}\right|^{1 / p}+C \delta\right) \leq \alpha+\omega_{h}\left(C\left|M_{\alpha}\right|\right) .
\end{aligned}
$$

Hence, (4.12) follows. Here, we have used the fact that the continuity of $y(x ; u(\cdot))$ in $x$ is uniform in $u(\cdot) \in \mathcal{U}($ see $(2.4))$.

Lemma 4.5. Let $(H)$ hold. Then, for any sequence $\left(y_{r}(\cdot), u_{r}(\cdot)\right) \in \mathcal{A}$ with

$$
\lim _{r \rightarrow \infty} d\left(y_{r}(\cdot), Q\right)=0
$$

it holds

$$
\varliminf_{r \rightarrow \infty} J_{r}\left(u_{r}(\cdot)\right) \geq \bar{m} .
$$

Proof. Suppose (4.23) is not the case. Then, for some $\varepsilon>0$ and some subsequence (still denoted by itself) $\left(y_{r}(\cdot), u_{r}(\cdot)\right) \in \mathcal{A}_{Q}$, we have

$$
\left(\int_{\Omega} h\left(x, y_{r}(x), u_{r}(x)\right)^{r} d x\right)^{1 / r} \leq \bar{m}-2 \varepsilon, \quad \forall r \geq r_{0} .
$$

Let

$$
\widehat{M}_{r}=\left\{x \in \Omega \mid h\left(x, y_{r}(x), u_{r}(x)\right)>\bar{m}-\varepsilon\right\} .
$$

Then,

$$
\bar{m}-2 \varepsilon \geq\left(\int_{\Omega} h\left(x, y_{r}(x), u_{r}(x)\right)^{r} d x\right)^{1 / r} \geq(\bar{m}-\varepsilon)\left|\widehat{M}_{r}\right|^{1 / r} .
$$

Thus,

$$
\left|\widehat{M}_{r}\right| \leq\left(\frac{\bar{m}-2 \varepsilon}{\bar{m}-\varepsilon}\right)^{r} \rightarrow 0, \quad(r \rightarrow \infty) .
$$


On the other hand, by Lemma 4.4 , there exists a $\left(\widetilde{y}_{r}(\cdot), \widetilde{u}_{r}(\cdot)\right) \in \mathcal{A}$, such that

$$
h\left(x, \widetilde{y}_{r}(x), \widetilde{u}_{r}(x)\right) \leq \bar{m}-\varepsilon+\widehat{\omega}\left(\left|\widehat{M}_{r}\right|\right)
$$

and

$$
\left|\left\{x \in \Omega \mid u_{r}(x) \neq \widetilde{u}_{r}(x)\right\}\right| \leq\left|\widehat{M}_{r}\right| \rightarrow 0
$$

Thus, Corollary 4.2 tells us that

$$
\begin{aligned}
d\left(\widetilde{y}_{r}(\cdot), Q\right) & \leq C\left\|y_{r}(\cdot)-\widetilde{y}_{r}(\cdot)\right\|_{W^{2, p}(\Omega)}+d\left(y_{r}(\cdot), Q\right) \\
& \leq C\left|\widehat{M}_{r}\right|^{1 / p}+d\left(y_{r}(\cdot), Q\right) \rightarrow 0
\end{aligned}
$$

Hence, by $(\mathrm{H})$, we obtain

$$
\varliminf_{r \rightarrow \infty} \operatorname{limssup}_{x \in \Omega} h\left(x, \tilde{y}_{r}(x), \tilde{u}_{r}(x)\right) \geq \bar{m}
$$

This contradicts (4.28). Thus, (4.23) holds.

Proof of Theorem 4.3. First of all, we have (recall $|\Omega|=1$ )

$$
J_{r}(u(\cdot)) \leq J(u(\cdot)), \quad \forall u(\cdot) \in \mathcal{U}
$$

Thus, it follows that

$$
\varlimsup_{r \rightarrow \infty} m_{r} \leq \bar{m}
$$

On the other hand, there exists $\left(y_{r}(\cdot), u_{r}(\cdot)\right) \in \mathcal{A}_{Q}$, such that

$$
J_{r}\left(u_{r}(\cdot)\right) \leq m_{r}+\frac{1}{r}, \quad r \geq 1 .
$$

Noting that $y_{r}(\cdot) \in Q$, by Lemma 4.5, we have

$$
\varliminf_{r \rightarrow \infty} J_{r}\left(u_{r}(\cdot)\right) \geq \bar{m}
$$

Hence, our conclusion follows from (4.33) and (4.35).

The idea of the proof for above result is taken from [3]. We have seen that assumption (H) plays an important role. 


\section{$\S 5$. Proof of Theorem 3.1.}

In this section we present a proof of Theorem 3.1. We first assume $h$ depends on $u$.

Let $\Omega_{0} \neq \emptyset$ (otherwise, there is nothing to prove). We introduce the so-called Ekeland distance in $\mathcal{U}$ :

$$
\widehat{d}(u(\cdot), \widehat{u}(\cdot))=|\{x \in \Omega \mid u(x) \neq \widehat{u}(x)\}|, \quad \forall u(\cdot), \widehat{u}(\cdot) \in \mathcal{U}
$$

From $[8,11]$, we know that $(\mathcal{U}, \widehat{d})$ is a complete metric space. Now, for $r>1$, we define

$$
\begin{gathered}
F_{r}(u(\cdot))=\left\{\left[\left(J_{r}(u(\cdot))-m_{r}+\frac{1}{r}\right)^{+}\right]^{2}+d_{Q}(y(\cdot ; u(\cdot)))^{2}\right\}^{1 / 2}, \\
\forall u(\cdot) \in \mathcal{U} .
\end{gathered}
$$

Then, we see that

$$
\left\{\begin{aligned}
F_{r}(u(\cdot)) & >0, \quad \forall u(\cdot) \in \mathcal{U}, \\
F_{r}(\bar{u}(\cdot)) & =\left(J_{r}(\bar{u}(\cdot))-m_{r}+\frac{1}{r}\right)^{+} \\
& \leq\left|J_{r}(\bar{u}(\cdot))-\bar{m}\right|+\left|\bar{m}-m_{r}\right|+\frac{1}{r} \equiv \sigma_{r} \rightarrow 0 .
\end{aligned}\right.
$$

Here, we have used Theorem 4.3. Thus, by Ekeland variational principle, we can find a $u_{r}(\cdot) \in \mathcal{U}$, such that

$$
\left\{\begin{array}{l}
d\left(u_{r}(\cdot), \bar{u}(\cdot)\right) \leq \sqrt{\sigma_{r}}, \\
F_{r}\left(u_{r}(\cdot)\right) \leq F_{r}(\bar{u}(\cdot)), \\
-\sqrt{\sigma_{r}} \widehat{d}\left(u_{r}(\cdot), \widehat{u}(\cdot)\right) \leq F_{r}(\widehat{u}(\cdot))-F_{r}\left(u_{r}(\cdot)\right), \quad \forall \widehat{u}(\cdot) \in \mathcal{U} .
\end{array}\right.
$$

Next, we let $s>0$ such that (noting $\Omega_{0} \neq \emptyset$ )

$$
\Omega_{s} \equiv\{x \in \Omega \mid h(x, \bar{y}(x), \bar{u}(x)) \leq \bar{m}-s\} \neq \emptyset
$$

Then, we let

$$
U_{s}(x)=\{u \in U \mid h(x, \bar{y}(x), u) \leq \bar{m}-s\}
$$

and define

$$
\Gamma_{s}(x)=\left\{\begin{array}{lc}
U_{s}(x), & x \in \Omega_{s} \\
U, & x \in \Omega \backslash \Omega_{s}
\end{array}\right.
$$


Then, it is clear that $\Gamma_{s}: \Omega \rightarrow 2^{U}$ is measurable and takes closed set values. Thus, by Filippov's lemma $([10])$, there exists a measurable selection $v(\cdot) \in \mathcal{U}$ with $v(x) \in$ $\Gamma_{s}(x)$, a.e. $x \in \Omega$. Then, we let $\mathcal{V}_{s}$ be the set of all such selections and let $\mathcal{U}_{s}^{r}$ be the set of all $u(\cdot) \in \mathcal{U}$ given by

$$
u(x)=\left\{\begin{array}{l}
v(x), \quad x \in \Omega_{s}, \\
u_{r}(x), \quad x \in \Omega \backslash \Omega_{s},
\end{array}\right.
$$

with $v(\cdot) \in \mathcal{V}_{s}$. Next, we fix any $u(\cdot) \in \mathcal{U}_{s}^{r}$. For any $\rho \in(0,1)$, we make a spike variation to the control: Let $E_{\rho} \subset \Omega$ be undetermined satisfying

$$
\left|E_{\rho}\right|=\rho|\Omega|=\rho
$$

and define

$$
u_{r}^{\rho}(x)=\left\{\begin{array}{l}
u_{r}(x), \quad x \in \Omega \backslash E_{\rho} \\
u(x), \quad x \in E_{\rho} .
\end{array}\right.
$$

Thus, $u_{r}^{\rho}(\cdot)$ is obtained by changing the values of $u_{r}(\cdot)$ only on the set $E_{\rho}$. Due to the fact that there is no convexity for $U$, only such perturbations are allowed. Then, we see that

$$
\widehat{d}\left(u_{r}(\cdot), u_{r}^{\rho}(\cdot)\right) \leq\left|E_{\rho}\right| \leq \rho .
$$

We let $y_{r}(\cdot)$ and $y_{r}^{\rho}(\cdot)$ are states corresponding to the controls $u_{r}(\cdot)$ and $u_{r}^{\rho}(\cdot)$, respectively. From [21] (see [11] also), we know that there exists a set $E_{\rho} \subset \Omega$ satisfying (5.8), such that the following hold:

$$
\left\{\begin{array}{l}
y_{r}^{\rho}(\cdot)=y_{r}(\cdot)+\rho z_{r}(\cdot)+\theta_{r}^{\rho}(\cdot), \\
\left\|\theta_{r}^{\rho}(\cdot)\right\|_{W^{1, p}(\Omega)}=o(1), \quad(\rho \rightarrow 0),
\end{array}\right.
$$

and

$$
\begin{gathered}
\int_{\Omega}\left(1-\frac{1}{\rho} \chi_{E_{\rho}}(x)\right)\left[h\left(x, y_{r}(x), u(x)\right)^{r}-h\left(x, y_{r}(x), u_{r}(x)\right)^{r}\right] d x=o(1) \\
(\rho \rightarrow 0)
\end{gathered}
$$

where $z_{r}(\cdot)$ is the solution of the following problem:

$$
\left\{\begin{aligned}
-\Delta z_{r}(x)= & f_{y}\left(x, y_{r}(x), u_{r}(x)\right) z_{r}(x) \\
& f\left(x, y_{r}(x), u(x)\right)-f\left(x, y_{r}(x), u_{r}(x)\right), \quad \text { in } \Omega \\
\left.z_{r}\right|_{\partial \Omega}= & 0 .
\end{aligned}\right.
$$


By (5.3), we see that as $r \rightarrow \infty$,

$$
\begin{array}{ll}
z_{r}(\cdot) \stackrel{w}{\rightarrow} z(\cdot), & \text { in } W^{2, p}(\Omega), \\
z_{r}(\cdot) \stackrel{s}{\rightarrow} z(\cdot), & \text { in } C(\bar{\Omega}),
\end{array}
$$

with $z(\cdot)$ being the solution of

$$
\left\{\begin{aligned}
-\Delta z(x)= & f_{y}(x, \bar{y}(x), \bar{u}(x)) z(x) \\
f(x, \bar{y}(x), u(x))-f(x, \bar{y}(x), \bar{u}(x)), \quad \text { in } \Omega, & \\
\left.z\right|_{\partial \Omega}= & 0 .
\end{aligned}\right.
$$

Now, taking $\widehat{u}(\cdot)=u_{r}^{\rho}(\cdot)$ in $(5.3)$, we obtain (note $(5.10)$ )

$$
\begin{aligned}
& -\sqrt{\sigma_{r}} \leq \frac{F_{r}\left(u_{r}^{\rho}(\cdot)\right)-F_{r}\left(u_{r}(\cdot)\right)}{\rho} \\
& \rightarrow \frac{1}{F_{r}\left(u_{r}(\cdot)\right)}\left\{( J _ { r } ( u _ { r } ( \cdot ) ) - m _ { r } + \frac { 1 } { r } ) ^ { + } \int _ { \Omega } \left(\frac{h_{r}(x)^{r-1}}{J_{r}\left(u_{r}(\cdot)\right)^{r-1}} \widehat{h}_{r}(x) z_{r}(x)\right.\right. \\
& \left.\left.\quad+\frac{\delta h_{r}(x)}{r J_{r}\left(u_{r}(\cdot)\right)^{r-1}}\right) d x+d_{Q}\left(y_{r}(\cdot)\right)\left\langle\nabla d_{Q}\left(y_{r}(\cdot)\right), z_{r}(\cdot)\right\rangle\right\} \\
& =\psi_{r}^{0} \int_{\Omega}\left(\mu_{r}(x) \widehat{h}_{r}(x) z_{r}(x)+\frac{\delta h_{r}(x)}{r J_{r}\left(u_{r}(\cdot)\right)^{r-1}}\right) d x+\left\langle\varphi_{r}, z_{r}(\cdot)\right\rangle,
\end{aligned}
$$

where

$$
\left\{\begin{array}{l}
h_{r}(x)=h\left(x, y_{r}(x), u_{r}(x)\right), \\
\widehat{h}_{r}(x)=g_{y}\left(x, y_{r}(x)\right), \\
\delta h_{r}(x)=h\left(x, y_{r}(x), u(x)\right)^{r}-h\left(x, y_{r}(x), u_{r}(x)\right)^{r},
\end{array}\right.
$$

and

$$
\left\{\begin{array}{l}
\psi_{r}^{0}=\frac{\left(J_{r}\left(u_{r}(\cdot)\right)-m_{r}+\frac{1}{r}\right)^{+}}{F_{r}\left(u_{r}(\cdot)\right)}, \\
\mu_{r}(x)=\frac{h_{r}\left(x, u_{r}(x)\right)^{r-1}}{\left\|h_{r}\left(\cdot, u_{r}(\cdot)\right)\right\|_{L^{r}(\Omega)}^{r-1}}, \\
\varphi_{r}=\frac{d_{Q}\left(y_{r}(\cdot)\right) \nabla d_{Q}\left(y_{r}(\cdot)\right)}{F_{r}\left(u_{r}(\cdot)\right)} .
\end{array}\right.
$$

We should note that $\varphi_{r}$ is always defined. It is clear that $([11,21])$

$$
\left(\psi_{r}^{0}\right)^{2}+\left\|\varphi_{r}\right\|_{\mathcal{Y}^{*}}^{2}=1 .
$$


The function $\mu_{r}(x) \geq 0$ satisfies (note $(2.16)$ )

$$
\begin{aligned}
& \int_{\Omega} \mu_{r}(x) d x \geq \int_{\Omega} \frac{h_{r}\left(x, u_{r}(x)\right)^{r}}{\left\|h_{r}\left(\cdot, u_{r}(\cdot)\right)\right\|_{L^{r}(\Omega)}^{r-1}} d x \\
& =\left\|h\left(\cdot, y_{r}(\cdot), u_{r}(\cdot)\right)\right\|_{L^{r}(\Omega)} \geq a, \quad \forall r>1,
\end{aligned}
$$

and

$$
\int_{\Omega} \mu_{r}(x) d x \leq\left\|\mu_{r}(\cdot)\right\|_{L^{r /(r-1)}(\Omega)}=1, \quad \forall r>1
$$

Then, we may assume

$$
\mu_{r}(\cdot) \stackrel{*}{\rightarrow} \mu, \quad \text { in }\left(L^{\infty}(\Omega)\right)^{*},
$$

for some $\mu \in\left(L^{\infty}(\Omega)\right)^{*}$. By (5.2)-(5.3), we have

$$
\lim _{r \rightarrow \infty} F_{r}\left(u_{r}(\cdot)\right)=0
$$

Thus, it follows from the definition of $F_{r}(u(\cdot))$ that

$$
\varlimsup_{r \rightarrow \infty} J_{r}\left(u_{r}(\cdot)\right) \leq \bar{m}
$$

Then, combining with Lemma 4.5 and (5.3), we see that

$$
\lim _{r \rightarrow \infty} J_{r}\left(u_{r}(\cdot)\right)=\bar{m}
$$

Hence, by the definition of $u(\cdot)$ (see $(5.7)$ ), we obtain

$$
\begin{aligned}
\int_{\Omega} \frac{\delta h_{r}(x)}{r J_{r}\left(u_{r}(\cdot)\right)^{r-1}} d x & \leq \frac{J_{r}\left(u_{r}(\cdot)\right)}{r} \int_{\Omega_{s}}\left(\frac{h\left(x, y_{r}(x), v(x)\right)}{J_{r}\left(u_{r}(\cdot)\right)}\right)^{r} d x \\
& \leq \frac{b}{r} \int_{\Omega_{s}}\left(\frac{h(x, \bar{y}(x), v(x))+\varepsilon_{r}}{\bar{m}-\varepsilon_{r}}\right)^{r} d x \\
& \leq \frac{b}{r} \int_{\Omega}\left(\frac{\bar{m}-s+\varepsilon_{r}}{\bar{m}-\varepsilon_{r}}\right)^{r} d x \rightarrow 0,
\end{aligned}
$$

where $\varepsilon_{r} \rightarrow 0$. On the other hand, by the convexity of $Q$, we have

$$
\left\langle\varphi_{r}, q(\cdot)-z_{r}(\cdot)\right\rangle \leq 0, \quad \forall q(\cdot) \in Q
$$


Thus, combining (5.14), (5.16), (5.25)-(5.27), we have (It is here that we need $h_{y}=g_{y}$ to be independent of $u$ !)

$$
\begin{gathered}
-\delta_{r} \leq \psi_{r}^{0}\left\langle\mu, g_{y}(\cdot, \bar{y}(\cdot)) z(\cdot)\right\rangle+\left\langle\varphi_{r}, z(\cdot)-(q(\cdot)-\bar{y}(\cdot))\right\rangle, \\
\forall z(\cdot) \in \mathcal{R}_{s}, \quad q(\cdot) \in Q,
\end{gathered}
$$

with $\delta_{r} \rightarrow 0$ as $r \rightarrow \infty$. Here, $\mathcal{R}_{s}$ is the reachable set of $(3.3)$ with $u(\cdot) \in \mathcal{U}_{s}$. Hence, by the finite codimensionality of $Q$, similar to [21], we may let

$$
\left\{\begin{array}{l}
\psi_{r}^{0} \rightarrow \bar{\psi}^{0}, \\
\varphi_{r} \stackrel{w}{\rightarrow} \bar{\varphi}, \quad \text { in } \mathcal{Y}^{*}
\end{array}\right.
$$

for some $\left(\bar{\psi}^{0}, \bar{\varphi}\right) \in[0,1] \times \mathcal{Y}^{*} \backslash\{0\}$. Thus, we take limits in (5.27) to obtain the transversality condition (3.8). Next, we take limits in $(5.28)$ with $q(\cdot)=\bar{y}(\cdot)$ to obtain

$$
0 \leq \bar{\psi}^{0}\left\langle\mu, g_{y}(\cdot, \bar{y}(\cdot)) z(\cdot)\right\rangle+\langle\bar{\varphi}, z(\cdot)\rangle, \quad \forall z(\cdot) \in \mathcal{R}_{s}
$$

Then, we let

$$
\psi^{0}=-\bar{\psi}^{0}, \quad \varphi=-\bar{\varphi}
$$

which changes (5.30) to the following

$$
0 \geq \psi^{0}\left\langle\mu, g_{y}(\cdot, \bar{y}(\cdot)) z(\cdot)\right\rangle+\langle\varphi, z(\cdot)\rangle, \quad \forall z(\cdot) \in \mathcal{R}_{s}
$$

We let $\psi_{r}(\cdot) \in W_{0}^{1, p^{\prime}}(\Omega)$ be the solution of

$$
\left\{\begin{array}{l}
-\Delta \psi_{r}(x)=f_{y}\left(x, y_{r}(x), u_{r}(x)\right) \psi_{r}(x)-\psi_{r}^{0} \mu_{r} g_{y}\left(x, y_{r}(x)\right)-\varphi_{r}, \quad x \in \Omega \\
\left.\psi_{r}\right|_{\partial \Omega}=0 .
\end{array}\right.
$$

By $[1,10,16]$,

$$
\left\|\psi_{r}(\cdot)\right\|_{W^{1, p^{\prime}}(\Omega)} \leq C\left(\left\|\mu_{r}\right\|_{L^{1}(\Omega)}+\left\|\varphi_{r}\right\| \mathcal{Y}^{*}\right) \leq C .
$$

Then, we may let

$$
\begin{cases}\psi_{r} \stackrel{w}{\rightarrow} \psi, & \text { in } W^{1, p^{\prime}}(\Omega) \\ \psi_{r} \stackrel{s}{\rightarrow} \psi, & \text { in } L^{p^{\prime}}(\Omega)\end{cases}
$$


Clearly, $\psi(\cdot)$ is the solution of (3.5). Then, combining (5.32) with some straightforward computations, we can obtain

$$
0 \geq \int_{\Omega} \psi(x)[f(x, \bar{y}(x), v(x))-f(x, \bar{y}(x), \bar{u}(x))] d x, \quad \forall v(\cdot) \in \mathcal{V}_{s}, \quad s>0
$$

Thus, the maximum condition (3.9) follows. Now, we show (3.6). If it is not the case, then, there exists a set $S \subset \Omega_{0}$, such that

$$
\mu(S) \equiv\left\langle\mu, \chi_{S}(\cdot)\right\rangle>0
$$

and for some $\varepsilon>0, r_{0} \geq 1$,

$$
h\left(x, y_{r}(x), u_{r}(x)\right) \leq \bar{m}-2 \varepsilon, \quad x \in S, \quad \forall r \geq r_{0}
$$

On the other hand, for $r$ large enough, one has

$$
\left\|h\left(\cdot, y_{r}(\cdot), u_{r}(\cdot)\right)\right\|_{L^{r}(\Omega)} \geq \bar{m}-\varepsilon
$$

Hence,

$$
\mu(S)=\lim _{r \rightarrow \infty} \int_{S} \mu_{r}(x) d x \leq\left(\frac{\bar{m}-2 \varepsilon}{\bar{m}-\varepsilon}\right)^{r-1}|S| \rightarrow 0
$$

This contradicts (5.37). Thus, (3.6) holds. Finally, from (5.20) we see that (3.7) holds.

In the case $h$ is independent of $u, \delta h_{r}=0$ and we can carry out the proof without considering $\Omega_{s}$ and $\mathcal{U}_{s}$ etc. Thus, the final conclusion of Theorem 3.1 follows.

\section{References}

[1] V. Barbu, Optimal Control of Variational Inequalities, Pitman, Boston, 1984.

[2] E. N. Barron, The Pontryagin maximum principle for minimax problems of optimal control, Nonlinear Anal., 15 (1990), 1155-1165.

[3] E. N. Barron and H. Ishii, The Bellman equation for minimizing the maximum cost, Nonlinear Anal., 13 (1989), 1067-1090.

[4] L. D. Berkovitz, Optimal Control Theory, Springer-Verlag, New York, 1974. 
[5] J. F. Bonnans and E. Casas, Optimal control of semilinear multistate systems with state constraints, SIAM J Control \& Optim., 27 (1989), 446-455.

[6] J. F. Bonnans and E. Casas, Un principe de Pontryagine pour le contrôle des systemes semilinearires elliptiques, J. Diff. Equ., 90 (1991), 288-303.

[7] E. Casas L. A. Fernandez, Optimal control of semilinear elliptic equations with pointwise constraints on the gradient of the state, preprint.

[8] I. Ekeland,, Nonconvex minimization problems, Bull. Amer. Math. Soc. (New Series), 1(1979), 443-474.

[9] D. Gilbarg and N. S. Trudinger, Elliptic Partial Differential Equations of Second Order, 2nd Edition, Springer-Verlag, 1983.

[10] C. J. Himmelberg, M. Q. Jacobs and F. S. Van Vleck, Measurable multifunctions, selectors and Filippov's implicit functions lemma, J. Math. Anal. Appl., 25 (1969), 276-284.

[11] X. Li and J. Yong, Necessary conditions of optimal control for distributed parameter systems, SIAM J. Control \& Optim., 29 (1991), 895-908.

[12] J. L. Lions, Optimal Control of Systems Governed by Partial Differential Equations, Springer-Verlag, New York, 1971

[13] C. Miranda, Partial Differential Equations of Elliptic Types, Springer-Verlag, New York, 1970.

[14] L. Neustadt, Optimization, Princeton Univ. Press, NJ, 1976.

[15] L. S. Pontryagin, V. G. Boltyanskii, R. V. Gamkrelidze and E. F. Mischenko, Mathematical Theory of Optimal Processes, Wiley, New York, 1962.

[16] G. Stampacchia, Le problème de Dirichlet pour les equations elliptiques du second ordre à coefficients discontinus, Ann. Inst. Fourier Grenoble, 15 (1965), 189-258.

[17] G. M. Troianiello, Elliptic Differential Equations and Obstacle Problems, Plenum Press, New York, 1987. 
[18] Y. Yao, Optimal control for a class of elliptic equations, Control Theory \& Appl., 1 (1984) No.3, 17-23 (in Chinese).

[19] J. Yong, A maximum principle for the optimal controls for a nonsmooth semilinear evolution system, Analysis and Optimization of Systems, A. Bensoussan and J. L. Lions eds., Lecture Notes in Control \& Inform. Sci., Vol 144, Springer-Verlag, 1990, $559-569$.

[20] J. Yong, Existence theory for optimal control of distributed parameter systems, Kodai Math. J., 15 (1992), 193-220.

[21] J. Yong, Pontryagin maximum principle for second order partial differential equations and variational inequalities, Diff. Int. Equ., 5 (1992), 1307-1334. 